\title{
LA RED SOCIAL-PERSONAL EN TRANSICIÓN: CAMBIOS EN EL TIEMPO*
}

\section{The social-personal net in transition: changes in time}

\author{
María Piedad Rangel M.** \\ Mariana Alievi Mari***k
}

Recibido: 15 de febrero de 2007 • Revisado: 26 de marzo de 2007 • Aceptado: 30 de marzo de 2007

\section{Resumen}

Este artículo presenta el proceso de cambio, durante el primer año de curso universitario, en la red social-personal de estudiantes de tres cursos universitarios, dos de ciencias de la salud y uno de ciencia humanas. Se aplicó el mapa de redes personal-social de Sluzki (1997). La metodología utilizada fue la Socioanálisis Cibernética propuesta por Delgado y Gutiérrez (1995), que permite analizar el lugar que ocupan los nudos de la red y también estudiar las diferencias entre los mismos derivadas de las características de los estudiantes. Se observaron cambios estructurales y funcionales del mapa y se observó la importancia de espacios de reflexión para facilitar en los estudiantes la inserción en el contexto universitario.

\section{Palabras clave}

Red social-personal, transición ecológica, estudiantes universitarios.

* Artículo que hace parte de la Tesis de doctorado Conceptos, Prácticas y Metodología en Redes Sociales, orientada por los profesores Jorge Castellá Sarriera y Neuza María de Fátima Guareschi.

* María Piedad Rangel M. Psicóloga. Especialista en Intervención Sistémica de la Familia por la USTA - Colombia. Magíster en Psicología Social y de la Personalidad por la PUC-RS. Doctora en Psicología por la PUC-RS. Profesora de la Universidade Luterana do Brasil - Canoas. Dirección correspondencia: Rua Castro Alves, 19/201 Porto Alegre, RS. CEP 90430-131. E-mail: piarangelm@gmail.com. Forma parte del grupo de investigación Relaciones, redes y narrativas, clasificado en categoría A por Colciencias.

* Estudiante de VII semestre de Psicología de la Universidad Regional Integrada del Alto Uruguay y de las Misiones, Becaria de Iniciación Científica que colaboró con la colecta y sistematización de los datos. 


\section{Abstract}

This article presents the change process, during the first year of university course, in the social-personal net of students of three university courses, two of the health and one of the human sciences. The personal-social map of nets of Sluzki was applied (1997). The used methodology was the Cybernetic Socioanalysis proposal from Delgado and Gutiérrez (1995), that allows to analyze the place the nots occupy in the net and also to study the differences between them, decurrently of the students characteristics. Structural and functional changes of the map had been observed as well as the importance of reflection spaces to facilitate the students insertion to the university context.

\section{Key words}

Social network, ecological transition, university students.

\section{Introducción}

Ingresar a la universidad implica una serie de cambios no solo para los novatos sino para to dos aquellos con los cuales ellos se relacionan y con quienes establecen nuevas relaciones.

La universidad se caracteriza por ser uno de los microsistemas de desarrollo humano en los que el sujeto gana más espacio para la to ma de decisiones y para la construcción de su nueva identidad: como profesional, como ciudadano y como responsable por si mismo.

Una fracción de los alumnos que ingresan a las universidades, además de experimentar cambios en la cultura escolar (transición del colegio a la universidad), pasa también por un periodo de alejamiento físico de la familia por la necesidad de vivir en otras localidades (traslado geográfico-cultural). Se enfrenta a la reorganización y reconstrucción de la red social de amistades, de colegas y de comunidad y de los servicios que esta le ofrece. Debe adaptarse a las costumbres de otras culturas (ciudad) y micro-culturas (residencias universitarias, casas de albergue, etc.). Esta transición ecológica y los cambios consecuentes llevan al alumno a vivir situaciones muchas veces desconocidas y él no tiene herra- mientas apropiadas que se encarguen de las reacciones emocionales y comportamentales derivadas de ellas, alterando la percepción sobre su bienestar. Los objetivos de esta investigación fueron estudiar el cambio en la red social-personal de alumnos universitarios a lo largo del primer año del curso, verificar las diferencias en los componentes de la red social-personal en el primer mes de ingreso a la universidad y en el último mes del primeraño.

\section{Cultura y socialización}

La universidad es un contexto donde los estudiantes pasan buena parte de su día a día. Este contexto se torna organizador de sus acciones, quehaceres, pensamientos y relaciones. De este modo, podemos pensar la universidad, pero principalmente la vida universitaria, como productora de cultura y espacio para la socialización. La universidad es el lugar de encuentro de múltiples referencias y referentes culturales. Cada estudiante y cada profesor traen una historia de vida que en las relaciones permanentes y continuadas se impactan, se modifican, se implican.

Pensando la cultura como el medio natural de desarrollo humano y del vivir de los sujetos, productora de la 
conciencia del hombre y constituida por estos (Bajtin y Vygotsky, 1993), podemos entender la cultura como el lugar donde se desarrollan diversos selves (McGoldrick, 2003): el de estudiante, amigo, hijo-hija, padre-madre, trabajadorestudiante, vecino-residente-ciudadano. Estos selves se constituyen en los diversos contextos de la red social-personal que co-construyen cultura e identidad.

Entender la cultura implica la comprensión del mundo socio-histórico que está constituido de significados (Thompson, 1995) que se construyen a partir de una variedad de fenómenos e intereses compartidos. Así, podemos pensar que en la vida universitaria se establecen intereses y características propias de este contexto que podrán reflejar modos particulares de organización y producción cultural.

\section{Redes de apoyo y redes social-personales}

Diferentes autores han escrito y trabajado con el concepto de redes de apoyo. Entre ellos, aquellos que trabajaron el concepto relacionándolo con la salud, afirmando que relaciones sociales saludables y efectivas previenen y reducen los efectos negativos del stress. Este interés le abrió el camino a un nuevo campo en la investigación social, en los años 70, denominado apoyo social. A mediados de los años 70 los trabajos de Castel en 1974, Cobb en 1976 y Caplan en 1974 hacían referencia al apoyo social. Castel y Cobb relataron que el apoyo social protege a las personas de influencias negativas, físicas y psicológicas (Gracia, 1998).

Cobb (1997) considera el apoyo social como la información que le permite a una persona sentirse querida, cuidada, estimulada, valorizada y parte de una red de relaciones que mantiene obligaciones recíprocas. Para Caplan (1974), un sistema de apoyo implica un patrón duradero de vínculos sociales que protegen la integridad física y psicológica de las personas y les ofrece el feed-back necesario para favorecer su identidad y desempeño (Gracia, 1998).
En la década de los años 80, Wasserman y Danforth (1988) definieron el apoyo social como un fenómeno "familiar" y un lugar común de la interacción humana, que aparece de manera espontánea entre las personas. Más tarde, en la década de los años 90, el concepto se amplía y se consideran aspectos estructurales y funcionales de las redes sociales, siendo que los aspectos estructurales estarían dados en términos de las condiciones objetivas que rodean el proceso de apoyo social que, en este caso, seria una de las funciones de la red (Gracia, 1998).

Gracia (1998) enfatiza la importancia de diferenciar entre los conceptos de red social, apoyo social y red de apoyo. Dice que la red social se refiere a las características estructurales de las relaciones sociales; el apoyo social se refiere a las funciones que presta esa red, yal efecto sobre el bienestar de las personas; finalmente, el concepto de red de apoyo es más restringido y denomina el conjunto de relaciones que desempeñan funciones de apoyo. Como esta forma de interacción aparece en las diferentes comunidades, que son unidades geográficas, este tejido podría denominarse red de redes. Dentro del concepto de función de apoyo social existe un acuerdo entre los diferentes autores a respecto de tres funciones básicas fundamentales: apoyo emocional, apoyo instrumental y apoyo de información.

Cuando se investiga con comunidades y grupos sobre sus redes de apoyo es importante diferenciar entre el apoyo percibido y el apoyo recibido debido a que de esta manera se abarcan dos aspectos de este constructo: el cognitivo y el conductual o comportamental. El apoyo social se da en tres contextos, a saber, el más exterior o general consiste en las relaciones establecidas con la comunidad; el intermedio consiste en las redes a través de las cuales se tiene acceso a un número relativamente amplio de personas; finalmente, las relaciones íntimas y de confianza constituyen el último, más cercano al centro y significativo contexto de relaciones sociales (Lin y Ensel, 1986, citados por Gracia, 1998).

Este mismo concepto es contemplado por Sluzki (1997) cuando define la red social como un nivel de relación 
microscópico en el que ocurren todas las relaciones que una persona percibe como significativas y diferenciadas de la masa anónima que es la sociedad. Para este autor la red social está formada por la familia, amistades, relaciones de trabajo o estudio y relaciones con la comunidad. En el contexto microscópico Sluzki (1997) también diferencia tres niveles de relación que están dados en términos de vínculo, de afinidad y de cercanía entre las personas y constituyen el microsistema donde se dan las interacciones más significativas de las personas.

Podemos definir las redes sociales como un sistema abierto en permanente construcción individual y colectivamente. Utilizan el conjunto de relaciones que poseen una persona y un grupo, y son fuente de reconocimiento, de sentimiento de identidad, del ser, de la competencia, de la acción. Están relacionadas con los papeles desempeñados en las relaciones con otras personas y grupos sociales (Montero, 2003) constituyéndose en las prácticas sociales que en el cotidiano no se aprovechan en su totalidad (Rangel, 2003; Rangel y Sarriera, 2005).

La identidad es entendida aquí como el lugar en el que un sujeto se reconoce a si mismo (Linares, 1996), reconocimiento de si que se da a través de las narrativas y de las experiencias relacionales permitiendo una conciencia de interacción con el otro. A través de la narrativa, la identidad es todo lo que puede decirse de si mismo, llevando en consideración lo que los otros dicen que es posible ser (Johnston, 1973). De ese modo, identidad es un lugar internalizado de la cultura a través de la narrativa que sobre el sujeto producen él mismo y los otros en la interacción.

Así, estos conceptos presentados podrán contribuir para la comprensión del proceso de inserción de los nuevos alumnos en la cultura universitaria, así como proveen modelos para investigar las características de estos alumnos y entender su percepción de bienestar psicológico.

\section{Presentación y discusión de los resultados}

En esta investigación se propuso la metodología del Socioanálisis Cibernético de Delgado y Gutiérrez (1995) para estudiar las redes sociales. Para efectos de la investigación, la Red Social se compone de una estructura física, ontológica, y una función que ocurre, principalmente, a través de la comunicación; o sea, las redes sociales también tienen una dimensión lingüística, comunicacional.

El modelo metodológico del Socioanálisis Cibernético (Delgado y Gutiérrez, 1995) permite comprender las configuraciones de las redes sociales de los alumnos universitarios y las organizaciones con las cuales se relacionan, así como las funciones de esta red social yel proceso de transición ecológica del colegio a la universidad.

El socioanálsis cibernético se basa en la conjunción de una teoría de los sistemas auto-organizados y en las investigaciones físicas so bre las estructuras disipativas y el orden fluctuante. Delgado y Gutiérrez, (1995) proponen que el foco del socioanálisis cibernético sea la relación del sistema con el entorno.

Comprender las relaciones so ciales a la luz de la cibernética de segundo orden, e introducir el concepto de desorden y caos, es posible si nos concebimos como sistemas observantes que, para efectos de la investigación, permiten crear un contexto investigativo en el cual todos los participante somos actores - activo (acéptese la redundancia), dando paso a un abordaje de la realidad cooperativa.

Los sistemas observantes u observadores se refieren a la observación del universo, la cual implica a quien lo describe (observa) dando lugar a la necesidad de mirar al observador. Desde esta nueva perspectiva, se establece una relación entre el observador y sus observaciones, ya que sus descripciones hablan, en primer lugar, del mismo, siendo posible en consecuencia ser paradójico; tal seria el caso de decir "yo soy un mentiroso" (Von Foerster, citado por Ibáñez, Coord., 1998a). 
A partir de la aplicación del mapa de redes (Sluzki, 1997) y del cuestionario para indagar las funciones solicitadas a los miembros de la red social-personal, fueron realizados grupos focales con la intención de discutir las relaciones de los estudiantes con las personas e instituciones que hacen parte de su red personal-social y de ese modo, comprender como establecen los vínculos y cuales funciones se solicitan de cuales contextos de la red social-personal, así como discutir sobre el bienestar psicológico de los discentes.

De este modo, los grupos focales sivieron para discutir tópicos (Romero, 2000) sobre la estructuración del tiempo, en el curso 1 - psicología, el ritual de primiparada ${ }^{1}$ en el curso de enfermería y la percepción de exclusión en el curso - 2 - filosofía; facilitando la integración grupal y movilizando a insights (Guareschi, 1996)así como comprender las percepciones que los participantes tienen sobre eventos específicos que emergen en la discusión (Morgan, 1998).

En total participaron 61 estudiantes universitarios, de dos cursos de departamento de ciencias humanas: psicología y filosofía, y un curso del departamento de cien- cias de la salud: enfermería, durante su primer año de vida universitaria.

La Institución en la cual ser realizó está localizada en un municipio al norte del estado ${ }^{2}$, con una población estimada por el IBGE ${ }^{3}$ en 27, 902 habitantes, en el año de 2005 (IBGE, 2006). La región se dedica a la agricultura y a la pecuaria caracterizando así su fuente de renta. En los últimos años esta actividad se ha visto afectada por las sequías, lo cual ha llevado a dificultades económicas que se reflejan en todos los aspectos como educación, salud y poder adquisitivo. El municipio cuenta con tres instituciones públicas y once privadas de salud. Le educación formal esta concentrada en escuelas municipales y estatales de enseñanza básica, contando con dos colegios de enseñanza media. El número de estudiantes va disminuyendo a medida que se sube de grado escolar e solamente una fracción reducida llega a cursar estudios superiores (IBGE, 2006).

La tabla que se muestra a continuación muestra la distribución de los estudiantes según características socio-demográficas, para cada curso participante de la investigación:

Tabla 1. Datos sociodemográficos por cursos

\begin{tabular}{lccc}
\hline & Psicología & Filosofía & Enfermería \\
Total & 23 & 12 & 26 \\
Media de edad & 21 & 28,2 & $\mathbf{1 8 , 8}$ \\
Desviación standart & 6,73 & 16 & 2,55 \\
Sexo. Fem. & $\mathbf{9 1 ,} \mathbf{3 0} \%$ & $50 \%$ & $\mathbf{9 6 , \mathbf { 1 5 } \%}$ \\
Masc. & $8,69 \%$ & $50 \%$ & $3,85 \%$ \\
Vive Solo & $4,35 \%$ & $16,66 \%$ & $38,46 \%$ \\
Vive con los padres o familia nuclear constituida. & $43,50 \%$ & $8,33 \%$ & $26,92 \%$ \\
Mora família criada & $8,69 \%$ & $16,66 \%$ & $3,85 \%$ \\
Mora com outros & $\mathbf{4 3 , 5 0 \%}$ & $58,33 \%$ & $30,77 \%$ \\
Mora na cidade & $82,61 \%$ & $83,34 \%$ & $80,77 \%$ \\
Mora fora da cidade & $17,39 \%$ & $16,66 \%$ & $19,23 \%$ \\
Trabalha & $17,39 \%$ & $33,33 \%$ & $\mathbf{0 \%}$ \\
Segundo curso superior & $4,35 \%$ & $\mathbf{2 5 \%}$ & $3,85 \%$ \\
\hline
\end{tabular}

El ritual de primíparos es una costumbre cultural muy fuerte en el Brasil. Este consiste en prácticas de actividades que muchas veces llevan al estudiante a vejámenes físicos, psicológicos y de comportamientos de riesgo, dando como resultado, ocasionalmente la muerte de algunos iniciantes universitarios. Por ejemplo, se les quitan los zapatos y se les obliga a salir a pedir limosna, se les retiran los documentos hasta que acepten realizar ciertas penitencias. En otros casos se ha ocasionado la muerte por coma alcohólico, o ahogamiento en piscinas de campus universitarios.

2 Corresponde a lo que en otros países se conoce como departamento, o comarca.

3 Instituto Brasileño de Geografía y Estadística, en el último censo oficial. 
Desde la recolección de los datos demográficos fue posible establecer algunas características diferentes entre los grupos. Es interesante resaltar que los estudiantes del curso de enfermería prefieren vivir solos y en segundo lugar con otros, a pesar que es el grupo que tiene media con edad mas baja. De otro lado los cursos de ciencias humanas, psicología y filosofía, prefiere en su mayoría vivir con otros. El alto porcentaje de estudiantes del sexo femenino en enfermería y psicología y el balance de masculino y femenino en el curso de filosofía, cursos refleja una característica cultural por la escogencia de cursos superiores. Un dato interesante, que fue observado durante el levantamiento de estos datos, es que $80 \%$ de los estudiantes de psicología y filosofía, que vive fuera de la cuidad en que estudia, vive en casa de sus padres, mientras que los estudiantes de enfermería que viven fuera del municipio tienen familias constituidas y consecuentemente son cabeza de familia.

Sobre el porcentaje de estudiantes que trabaja fue observado que ninguno del curso de enfermería lo hace. En los cursos de psicología y filosofía se encontró estudiantes que trabajan. La diferencia entre los cursos del departamento de ciencias humanas, es que los del curso de psicología trabajan únicamente los fines de semana 0 algunas horas durante la misma y los del curso de filosofía tienen trabajo en tiempo integral. Esto tal vez pueda relacionarse con la edad promedio de estos estudiantes que es de 28, 2, con desviación Standard de 16 , indicando que estarían en el ciclo de vida personal o familiar en que una de las ficciones principales es el trabajo (Abengózar, Cerda y Meléndez, 1998). También se observó, en ese grupo, que $25 \%$ está estudiando el segundo curso superior, lo que ayuda a explicar la edad de los mismos.

En el primer encuentro con los grupos se explico que el trabajo a ser realizado tendría una duración de un año académico, durante el cual pretendíamos comprender todos, investigadora e investigados, la transición ecológica que consiste en un proceso por el cual pasan las personas cuando cambian de un contexto sociocultural para otro; la reorganización y reconstruc- ción de redes sociales-personales, porque todo cambio contextual implica una migración de miembros pertenecientes a esta red; el proceso de aculturación, que consiste en aprender las costumbres de otras culturas y microculturas, sin necesariamente, perder la de origen, logrando convivir con varias forma culturales; y, finalmente como consecuencia de estas discusiones se procuraba contribuir con esta vivencia universitaria de transición enfatizando la importancia del efecto de la intervención que provocan las formas diversas de investigación participante.

En el segundo encuentro se solicitó lectura atenta y posterior firma del Consentimiento Libre y Aclarado para responder a los instrumentos utilizados en la investigación, que indicaba que la investigación había sido evaluada y aceptada por el comité de Bioética de la Universidad Regional Integrada del Alto Uruguay y de las Misiones, mediante protocolo № 026 - 21PP-H05.

Se explicó el procedimiento de la actividad a ser realizada, la cual constaba de un Instrumento llamado Mapa de Redes (Sluzki, 1997) a ser construido a partir de las relaciones de cada uno. En el mapa deben ser colocadas las personas e instituciones percibidas por los estudiantes, discriminadas por cuadrantes de familia, amigos, universidad/trabajo y comunidad. El mapa, además de los cuadrantes, consta de tres círculos concéntricos en donde se ubican las personas e instituciones de acuerdo con la percepción de cercanía o distanciamiento de los vínculos relacionales. En seguida, deberían responder a un cuestionario que indagaba a quien eran solicitadas las funciones de la red. Se resaltó que la red social se refiere al conjunto de todas las relaciones que una persona percibe como significativas o define como diferenciadas de la masa anónima de la sociedad (Sluzki, 1997).

Después de trascurridos ocho meses de vida universitaria, en el quinto encuentro, fueron recordadas las instrucciones para responder al mapa de redes ya que este fue repetido en ese momento. Se explicó la importancia de utilizar los mismos instrumentos para verificar los cambios ocurridos en la rede social-personal del grupo. 
Enseguida se presentan, en tablas, los resultados de la primera y segunda aplicaciones del Mapa de redes, por cursos:

Tabla 2. Psicología. Número de miembros en la red según cuadrantes: familia, amigos, comunidad, trabajo/universidad. Percepción de cercanía o distanciamiento

(Hs. hombres, Ms. Mujeres; Inst. instituciones).

\begin{tabular}{|c|c|c|c|c|c|c|c|c|c|c|c|c|}
\hline \multirow[t]{2}{*}{$\begin{array}{l}\text { Primera } \\
\text { Aplicación }\end{array}$} & \multicolumn{3}{|c|}{ FAMILIA } & \multicolumn{3}{|c|}{ AMIGOS } & \multicolumn{3}{|c|}{ COMUNIDAD } & \multicolumn{3}{|c|}{ UNIV/ TRAB } \\
\hline & Hs. & Ms. & Inst. & Hs. & Ms. & Inst. & Hs. & Ms. & Inst. & Hs. & Ms. & Inst. \\
\hline Cercanía & 122 & 86 & 0 & 131 & 26 & 0 & 0 & 1 & 47 & 83 & 25 & 11 \\
\hline Intermediario & 89 & 66 & 0 & 72 & 48 & 0 & 1 & 5 & 20 & 50 & 16 & 11 \\
\hline Distante & 41 & 56 & 0 & 40 & 26 & 0 & 0 & 0 & 10 & 36 & 13 & 5 \\
\hline Subtotal & 252 & 208 & 0 & 243 & 100 & 0 & 1 & 6 & 77 & 169 & 54 & 27 \\
\hline Total & 460 & & & 343 & & & 84 & & & 250 & & \\
\hline Media & 20 & & & $14-15$ & & & $3-4$ & & & $10-11$ & & \\
\hline Densidad & $40,60 \%$ & & & $30,27 \%$ & & & $7,06 \%$ & & & $22.06 \%$ & & \\
\hline $\begin{array}{l}\text { Distribución y } \\
\text { composición }\end{array}$ & $100 \%$ P 0\% & & & $100 \%$ P 0\% & & & $8,33 \% \mathrm{P} 9$ & $66 \% 1$ & & 89 & $2 \%$ P 10 & $8 \% 1$ \\
\hline
\end{tabular}

Tabla 3. Psicología. Número de de miembros en la red según los cuadrantes: familia, amigos, comunidad, trabajo/ universidad. Percepción de cercanía o distanciamiento

(Hs. hombres, Ms. Mujeres; Inst. instituciones).

\begin{tabular}{|c|c|c|c|c|c|c|c|c|c|c|c|c|}
\hline \multirow[t]{2}{*}{$\begin{array}{l}\text { Segunda } \\
\text { Aplicación }\end{array}$} & \multicolumn{3}{|c|}{ FAMILIA } & \multicolumn{3}{|c|}{ AMIGOS } & \multicolumn{3}{|c|}{ COMUNIDAD } & \multicolumn{3}{|c|}{ UNIV/TRAB } \\
\hline & Hs. & Ms. & Inst. & Hs. & Ms. & Inst. & Hs. & Ms. & Inst. & Hs. & Ms. & Inst. \\
\hline Próximo & 84 & 55 & 0 & 106 & 35 & 0 & 30 & 13 & 27 & 75 & 15 & 15 \\
\hline Intermediario & 86 & 69 & 1 & 90 & 37 & 2 & 16 & 8 & 17 & 57 & 14 & 10 \\
\hline Distante & 79 & 64 & 3 & 41 & 32 & 1 & 18 & 14 & 4 & 42 & 10 & 4 \\
\hline Subtotal & 249 & 188 & 4 & 237 & 104 & 3 & 64 & 35 & 48 & 174 & 39 & 29 \\
\hline Total & 371 & 344 & 147 & 242 & & & & & & & & \\
\hline Media & $16-17$ & $14-15$ & $6-7$ & $10-11$ & & & & & & & & \\
\hline Densidad & \multicolumn{2}{|c|}{$33,60 \%$} & & \multicolumn{3}{|l|}{$31,15 \%$} & \multicolumn{3}{|l|}{$13,31 \%$} & \multicolumn{3}{|c|}{$21,92 \%$} \\
\hline \multicolumn{4}{|c|}{$\begin{array}{l}\text { Distribución } \\
\text { y composición } 98,9 \% \text { P- 1, 08\%। }\end{array}$} & \multicolumn{3}{|c|}{$99,12 \% \mathrm{P}-0,87 \% \mathrm{I}$} & \multicolumn{3}{|c|}{$67,34 \% P-32,65 \% I$} & \multicolumn{3}{|c|}{$88,02 \%$ P- $11,98 \%$ I } \\
\hline
\end{tabular}


Tabla 4. Filosofía. Número de miembros en la rede según los cuadrantes: familia, amigos, comunidad, trabajo/universidad. Percepción de proximidad o distanciamiento

(Hs. hombres, Ms. Mujeres; Inst. instituciones).

\begin{tabular}{|c|c|c|c|c|c|c|c|c|c|c|c|c|}
\hline \multicolumn{13}{|l|}{ Primera } \\
\hline \multirow[t]{2}{*}{ Aplicación } & \multicolumn{3}{|c|}{ FAMILIA } & \multicolumn{3}{|c|}{ AMIGOS } & \multicolumn{3}{|c|}{ COMUNIDAD } & \multicolumn{3}{|c|}{ UNIV/TRAB } \\
\hline & Hs. & Ms. & Inst. & Hs & Ms & Inst. & Hs. & Ms. & Inst. & Hs. & Ms. & Inst. \\
\hline Próximo & 82 & 52 & 0 & 168 & 86 & 0 & 15 & 26 & 36 & 58 & 52 & 18 \\
\hline Intermedio & 58 & 51 & 0 & 51 & 32 & 0 & 13 & 15 & 23 & 51 & 37 & 12 \\
\hline Distante & 69 & 77 & 0 & 44 & 29 & 0 & 4 & 9 & 10 & 1 & 7 & 11 \\
\hline Subtotal & 209 & 180 & 0 & 263 & 147 & 0 & 32 & 50 & 69 & 110 & 96 & 41 \\
\hline Total & 389 & & & 510 & & & 151 & & & 247 & & \\
\hline Media & $32-33$ & & & $42-43$ & & & $12-13$ & & & $20-21$ & & \\
\hline Densidad & $29.22 \%$ & & & $39.32 \%$ & & & $11.64 \%$ & & & $19.04 \%$ & & \\
\hline $\begin{array}{l}\text { Distribución } \\
\text { y composición }\end{array}$ & $100 \%$ P $0 \%$ & & & $100 \%$ P $0 \%$ & & & $54.30 \%$ & P $45.6 \mathrm{~S}$ & & $83.4 \% \mathrm{P} 1$ & $6.60 \% \mathrm{I}$ & \\
\hline
\end{tabular}

Tabla 5 Filosofía. Número de miembros en la rede según los cuadrantes: familia, amigos, comunidad, trabajo/universidad. Percepción de proximidad o distanciamiento

(Hs. hombres, Ms. Mujeres; Inst. instituciones).

\begin{tabular}{|c|c|c|c|c|c|c|c|c|c|c|c|c|}
\hline \multicolumn{13}{|l|}{ Segunda } \\
\hline \multirow[t]{2}{*}{ Aplicación } & \multicolumn{3}{|c|}{ FAMILIA } & \multicolumn{3}{|c|}{ AMIGOS } & \multicolumn{3}{|c|}{ COMUNIDAD } & \multicolumn{3}{|c|}{ UNIV/ TRAB } \\
\hline & Hs. & Ms. & Inst. & Hs & Ms & Inst. & Hs. & Ms. & Inst. & Hs. & Ms. & Inst. \\
\hline Próximo & 54 & 39 & 0 & 42 & 44 & 0 & 27 & 32 & 16 & 33 & 42 & 12 \\
\hline Intermedio & 35 & 41 & 0 & 53 & 46 & 0 & 32 & 28 & 6 & 24 & 26 & 11 \\
\hline Distante & 48 & 44 & 0 & 33 & 32 & 0 & 19 & 12 & 11 & 8 & 12 & 9 \\
\hline Subtotal & 137 & 124 & 0 & 128 & 122 & 0 & 78 & 72 & 33 & 65 & 80 & 32 \\
\hline Total & 261 & & & 250 & & & 183 & & & 177 & & \\
\hline Media & $21-22$ & & & $20-21$ & & & $15-16$ & & & $14-15$ & & \\
\hline Densidad & $30 \%$ & & & $28.70 \%$ & & & $21 \%$ & & & $20.30 \%$ & & \\
\hline \multicolumn{13}{|l|}{ Distribución } \\
\hline
\end{tabular}


Tabla 6 Enfermería. Número de miembros en la red según los cuadrantes: familia, amigos, comunidad, trabajo/universidad. Percepción de proximidad o distanciamiento

(Hs. hombres, Ms. Mujeres; Inst. instituciones).

\begin{tabular}{|c|c|c|c|c|c|c|c|c|c|c|c|c|}
\hline \multicolumn{13}{|l|}{ Primera } \\
\hline \multirow[t]{2}{*}{ Aplicación } & \multicolumn{3}{|c|}{ FAMILIA } & \multicolumn{3}{|c|}{ AMIGOS } & \multicolumn{3}{|c|}{ COMUNIDAD } & \multicolumn{3}{|c|}{ UNIV/ TRAB } \\
\hline & Hs. & Ms. & Inst. & $\mathrm{Hs}$ & Ms & Inst. & Hs. & Ms. & Inst. & Hs. & Ms. & Inst. \\
\hline Próximo & 38 & 25 & 0 & 55 & 29 & 0 & 6 & 3 & 14 & 50 & 25 & 6 \\
\hline Intermedio & 30 & 25 & 0 & 23 & 27 & 0 & 6 & 7 & 12 & 25 & 12 & 6 \\
\hline Distante & 25 & 19 & 0 & 21 & 21 & 0 & 3 & 3 & 4 & 12 & 13 & 0 \\
\hline Subtotal & 93 & 69 & 0 & 99 & 77 & 0 & 15 & 13 & 30 & 87 & 50 & 12 \\
\hline Total & 162 & & & 176 & & & 58 & & & 149 & & \\
\hline Media & $6-7$ & & & $6-7$ & & & $2-3$ & & & $5-6$ & & \\
\hline Densidad & $29,72 \%$ & & & $32,3 \%$ & & & $10,64 \%$ & & & $27,33 \%$ & & \\
\hline $\begin{array}{l}\text { Distribución y } \\
\text { composición }\end{array}$ & $100 \%$ P 0 & & & $100 \% \mathrm{PC}$ & & & $48.27 \%$ P 5 & $1,72 \%$ I & & $91,94 \% \mathrm{P}$ & $8,05 \%$ & \\
\hline
\end{tabular}

Tabla 7 Enfermería. Número de miembros en la rede según los cuadrantes: familia, amigos, comunidad, trabajo/universidad. Percepción de proximidad o distanciamiento

(Hs. hombres, Ms. Mujeres; Inst. instituciones).

\begin{tabular}{|c|c|c|c|c|c|c|c|c|c|c|c|c|}
\hline \multirow{3}{*}{$\begin{array}{l}\text { Segunda } \\
\text { Aplicación }\end{array}$} & \multirow{2}{*}{\multicolumn{3}{|c|}{ FAMILIA }} & \multirow{2}{*}{\multicolumn{3}{|c|}{ AMIGOS }} & \multirow{2}{*}{\multicolumn{3}{|c|}{ COMUNIDAD }} & \multirow{2}{*}{\multicolumn{3}{|c|}{ UNIV/TRAB }} \\
\hline & & & & & & & & & & & & \\
\hline & Hs. & Ms. & Inst. & Hs. & Ms. & Inst. & Hs. & Ms. & Inst. & Hs. & Ms. & Inst. \\
\hline Próximo & 63 & 59 & 2 & 111 & 57 & 1 & 21 & 18 & 41 & 88 & 46 & 31 \\
\hline Intermediario & 68 & 63 & 0 & 110 & 63 & 0 & 10 & 11 & 13 & 92 & 19 & 20 \\
\hline Distante & 45 & 40 & 0 & 54 & 48 & 0 & 9 & 8 & 8 & 42 & 11 & 4 \\
\hline Subtotal & 176 & 162 & 2 & 275 & 168 & 1 & 40 & 37 & 62 & 222 & 76 & 55 \\
\hline Total & 340 & 444 & 139 & 353 & & & & & & & & \\
\hline Media & \multicolumn{2}{|l|}{$13-14$} & \multicolumn{4}{|c|}{$17-18$} & \multicolumn{3}{|l|}{$5-6$} & \multicolumn{3}{|l|}{$13-14$} \\
\hline Densidad & \multicolumn{3}{|c|}{$26,64 \%$} & \multicolumn{3}{|c|}{$34,8 \%$} & \multicolumn{3}{|l|}{$10,9 \%$} & \multicolumn{3}{|c|}{$27,66 \%$} \\
\hline $\begin{array}{l}\text { Distribución y } \\
\text { composición }\end{array}$ & \multicolumn{3}{|c|}{$99 \%$ P $1 \%$ I } & & \multicolumn{2}{|c|}{$99 \%$ P $1 \%$ I } & \multicolumn{3}{|c|}{$55,39 \%$ P 44, 6\%। } & \multicolumn{3}{|c|}{$84,41 \%$ P $15,58 \%$ I } \\
\hline
\end{tabular}


En las tablas 2 a 7 se presentan los totales de los componentes de la red social-personal de los estudiantes, por cursos, en la primera y segunda aplicaciones del Mapa de Redes, que representa la dimensión estructural de la red, que evalúa tamaño, densidad, composición y distribución y homogeneidad - heterogeneidad.

Referente al tamaño de la red, se aprecia que en el curso de psicología la media por estudiante, de la red social-personal total es de 49 a 50 miembros en la primera aplicación y de 48 en la segunda, no siendo observado cambio significativo (tablas 2 y 3 ). Podemos notar que se mantuvo constante el número total, pero hubo un desplazamiento de integrantes de la red, disminuyendo en el cuadrante de familia y aumentando en el de la comunidad. Se mantuvo estable en el cuadrante de amigos y de universidad - trabajo. En las discusiones con los estudiantes fue explicitado que, en el transcurso el primer año, estos redefinieron la percepción sobre la Comunidad, pasando a percibir la importancia de esta en sus vidas. Esto puede ser consecuencia de las características del curso, cuyo objetivo es trabajar con el otro.

En el curso de filosofía, la media total para la red socialpersonal fue de 108 a 109 miembros en la primera aplicación y de 72 a 73 en la segunda (tablas 4 y 5). Siendo notada una disminución considerable en la media, fue discutido con los estudiantes este hecho y la explicación fue que uno de los estudiantes, cuando fue realizada la primera aplicación, dijo haber evaluado inadecuadamente el concepto de amistan, colocando también conocidos e integrantes de una congregación religiosa, pero que, en la segunda aplicación, había dejado todos estos fuera de su mapa. Esta situación se explica debido a las características del curso referido, ya que muchos de los estudiantes son seminaristas y las mujeres pertenecen a congregaciones religiosas, que tienen sistemas de creencias de hermandad y asociación. Una vez considerada esta situación particular, se observó un aumento en el cuadrante de la Comunidad lo que se entiende también por el envolvimiento de las actividades de los estudiantes en este contexto.
En el curso de enfermería la media en la primera aplicación fue de 20 a 21 miembros y en la segunda fue de 49 a 50 miembros, observando un aumento de más de $100 \%$ (tablas 6 y 7). El aumento aconteció en todos los cuadrantes. Cuando cuestionados los estudiantes, estos respondieron que en I segunda aplicación habían pensado más atentamente sus relaciones y que, en el primer mes de universidad, cuando fue realizado el primer mapa, estaban asustados con la misma. Recordaron de nuevo el ingreso a la universidad y especialmente el ritual de primíparos como un estresor importante.

La red social se constituye de las relaciones percibidas por las personas (Rangel, 2004; Sluzki, 1997). Cuando en el contexto social se encuentran personas e instituciones que pueden desempeñar funciones de la red, pero pasan sin ser percibidas no se piensa estas como suporte social trayendo como consecuencia la imposibilidad de movilizar la red. El reconocimiento de su existencia es de fundamental importancia para solicitar el ejercicio de las funciones y para ampliar la posibilidad de obtener apoyo social de las necesidades personales.

En todos los cursos el número de miembros por cuadrante varía entre tres y 44 integrantes. De este modo, podemos entender que la red social-personal de los alumnos de los cursos estudiados presenta todas las características que Rangel (2004) y Sluzki) describen para la dimensión del tamaño de la red. Tres miembros por cuadrante san considerados insuficientes $y$, en el otro extremo, 44 se considera grande. Esto porque el tamaño medio es de 50 miembros o nodos el la totalidad de la red (Elkaïm, 1995). Estos autores señalan que el tamaño medio es el más aceptable puesto que este número facilita efectivar la red para dar soporte a las funciones solicitadas. Si la persona cuenta con una red pequeña o grande se producen efectos de red menos eficaces por cuanto en el primer caso cuando se necesita de alguna fusión específica puede darse el caso de no haber en la red persona 0 institución que tenga las condiciones de hacerlo, o si por el contrario la red es muy grande aparece la tendencia de que se pase para otro la acción de la ayuda. 
Asimismo, se observa que el cuadrante de la comunidad, en las dos aplicaciones, cuenta con el menor número de integrantes o nódulos de la red, mientras que los de familia y amigos son los cuadrantes que aportan mayor número de participantes. Entendemos, por lo tanto, que las funciones serán solicitadas y llevadas a cabo de modo mas eficaz cuando se pidan para amigos y familiares.

Sobre la densidad, en el curso de psicología se observó que el cuadrante de la familia fue el más denso es el en las dos aplicaciones. En lo que respecta al cuadrante de comunidad, se observo un incremento de casi $100 \%$ (7.06\% a 13.31\%). De otro lado, los cuadrantes de amigos y universidad/trabajo se mantuvieron estables (tablas 2 y 3). Entendemos que a lo largo del año académico, los alumnos lograron percibir el contexto comunitario, haciéndolo visible y, en consecuencia integrando nódulos provenientes de este, a su red social-personal.

En el curso de filosofía la densidad del cuadrantes de amigos disminuyó, por las razones expuestas anteriormente, de error de interpretación del mapa por parte de un estudiante. Sin embargo, es interesante observar que aconteció un aumento importante en el cuadrante de la comunidad, que puede explicarse de la misma forma que para el curso de psicología. Por otra parte los contextos de familia y universidad/trabajo mantuvieron su densidad estable (tablas 4 y 5 ).

En el curso de enfermería es el cuadrante de amigos el que obtiene la más alta densidad en las dos aplicaciones, lo que refleja que, $38,46 \%$ de estos estudiantes viviendo solos sienten la necesidad de una vinculación social más fuerte con amigos y colegas de universidad (tablas 6 y 7). Llama la atención que en este curso no se observó aumento en el cuadrante de la comunidad como en los cursos pertenecientes al departamento de ciencias humanas. Podemos entender que los cursos de ciencias humanas, desde los semestres iniciales llevan a los alumnos a pensar el lugar social que estos ocupan, permitiendo se reflexione y se visibilice la comunidad y el entorno social. Por otro lado los primeros semestres de ciencias de la salud, y específicamente enfermería tienen en su currículo materias más técnicas en el inicio de la carrera y entran en contacto con pacientes más adelante.

Sobre la distribución y composición, que se refiere a la presencia en la red de personas e instituciones (LópezCabanas, 1997; Sluzki, 1997), se observó en los cursos de psicología y filosofía que en el cuadrante de familias y amigos no aparecen instituciones (tablas 2, 3, 4 y 5). En el curso de enfermería aparece referencia a la percepción de instituciones en la segunda aplicación, pero en baja proporción (tablas 2 y 3). En el cuadrante de universidad/trabajo la distribución y composición se mantuvieron y en el de la comunidad la proporción cambió significativamente en todos los cursos en la segunda aplicación. Podemos inferir que, si en un primer momento (primera aplicación) la comunidad era vista principalmente a través de las instituciones como salud, asociaciones, recreación y diversión, en la segunda las personas dentro de estas instituciones cobraron visibilidad. Ya la comunidad no es más percibida solamente como un conjunto de servicio, sino como personas dentro de ellas que los viabilizan.

Una vez discutida la estructura de la red, fue preguntado a los estudiantes sobre la percepción del número de participantes en su red, para cada cuadrante. Los resultados se presentan en tablas yen seguida se hace la discusión de las funciones según las personas o instituciones en los cuadrantes a los cuales estas son solicitadas. 
Tabla 8 Psicología. Percepción de suficiencia o insuficiencia de los miembros en los diversos cuadrantes del mapa de redes social-personal.

\begin{tabular}{|c|c|c|c|c|c|c|c|c|}
\hline & \multicolumn{2}{|c|}{ FAMILIA } & \multicolumn{2}{|c|}{ AMIGOS } & \multicolumn{2}{|c|}{ COMUNIDAD } & \multicolumn{2}{|c|}{ UNIV/TRABALHO } \\
\hline Aplicación & $1 \underline{a}$ & $2 \underline{a}$ & $1^{\underline{a}}$ & $2^{a}$ & $1 \underline{a}$ & $2^{a}$ & $1 \underline{a}$ & $2 \underline{a}$ \\
\hline Suficiente & $88,88 \%$ & $95 \%$ & $59,25 \%$ & $65 \%$ & $33,33 \%$ & $40 \%$ & $29,63 \%$ & $35 \%$ \\
\hline Insuficiente & $11,11 \%$ & $5 \%$ & $40,74 \%$ & $35 \%$ & $66,66 \%$ & $60 \%$ & $70,47 \%$ & $65 \%$ \\
\hline
\end{tabular}

Tabla 9 Filosofía. Percepción de suficiencia o insuficiencia de los miembros en los diversos cuadrantes del mapa de redes social-personal.

\begin{tabular}{|c|c|c|c|c|c|c|c|c|}
\hline \multirow[b]{2}{*}{ Aplicación } & \multicolumn{2}{|c|}{ FAMILIA } & \multicolumn{2}{|c|}{ AMIGOS } & \multicolumn{2}{|c|}{ COMUNIDAD } & \multicolumn{2}{|c|}{ UNIV/TRABALHO } \\
\hline & $1 \underline{a}$ & $2 \underline{a}$ & $1 \underline{a}$ & $2^{a}$ & $1 \underline{a}$ & $2^{a}$ & $1 \underline{a}$ & $2^{a}$ \\
\hline Suficiente & $75 \%$ & $55 \%$ & $41,66 \%$ & $18 \%$ & $41,66 \%$ & $27 \%$ & $58,33 \%$ & $46 \%$ \\
\hline Insuficiente & $25 \%$ & $45 \%$ & $58.33 \%$ & $82 \%$ & $58,33 \%$ & $73 \%$ & $41,66 \%$ & $54 \%$ \\
\hline
\end{tabular}

Tabla 10 Enfermería. Percepción de suficiencia o insuficiencia de los miembros en los diversos cuadrantes del mapa de redes social-personal.

\begin{tabular}{lcccccccc} 
& \multicolumn{2}{c}{ FAMILIA } & \multicolumn{2}{c}{ AMIGOS } & \multicolumn{2}{c}{ COMUNIDAD } & \multicolumn{2}{c}{ UNIV/TRABALHO } \\
\hline Aplicación & $1 \underline{\underline{a}}$ & $2 \underline{\underline{a}}$ & $1 \underline{\underline{a}}$ & $2 \underline{\underline{a}}$ & $1 \underline{\underline{a}}$ & $2 \underline{\underline{a}}$ & $1 \underline{\underline{a}}$ & $2 \underline{\underline{a}}$ \\
Suficiente & $100 \%$ & $86 \%$ & $69 \%$ & $76 \%$ & $33 \%$ & $38 \%$ & $40 \%$ & $52 \%$ \\
Insuficiente & $0 \%$ & $14 \%$ & $31 \%$ & $24 \%$ & $67 \%$ & $62 \%$ & $60 \%$ & $48 \%$ \\
\hline
\end{tabular}

Se observa en las tablas 8, 9 y 10 que la percepción del número de participantes en el cuadrante de la familia fue el de mayor significancia en la primera y segunda aplicaciones en todos los cursos e inclusive, en el curso de psicología esta percepción se encontró aumentada. Para los cursos de filosofía y enfermería la percepción de suficiencia de este cuadrante, en la segunda aplicación, disminuyó. Esto fue explicado por los estudiantes por el hecho de que estos permanecen durante todo el año lejos de aquellas puesto que el curso estudiando, sumado a otras actividades de trabajo o formación, solamente facilitan el contacto directo con sus familias en las vacaciones de verano. Manifestaron que, cada vez más, sienten la distancia y nostalgia por la familia. Esto porque como señalan Berger y Luckman (1999), las interacciones cara a cara cobran para las personas el carácter de realidad. Es a través de ese tipo de interacción que se da el reconocimiento del otro y es posible apreciar el afecto, el reconocimiento y el amor (Brenson, 1981). Barman (2005) critica las formas modernas y 
posmodernas de las relaciones diciendo que la mediatización de las mismas y el poco tiempo dedicado a las relaciones, produce una inmediatez carente de valor. Inclusive considera este autor que las relaciones han venido perdiendo la carga afectiva y que encontramos, en la actualidad divorcios sin dolor, divorcios light. Esta crítica se evidencia en las discusiones que sobre relaciones se realizaron con los estudiantes; para este grupo, las relaciones familiares todavía se piensan a la moda "antigua". Ellos buscan la presencia física y el contacto visual.

En el cuadrante de amigos, en la segunda aplicación, la percepción de insuficiencia disminuyó significativamente en el curso de filosofía. Esta percepción puede entenderse debido a que en el transcurso del año fueron cambiando de amistades. Las interacciones con los antiguos compañeros de colegio y trabajo, del barrio, de las actividades recreativas fueron más esporádicas desde que iniciaron los estudios, y en ocasiones, se perdieron. A pesar de que el número de amigos aumentó, en el curso de enfermería, la percepción de pérdida se evidenció. Es lo que en la migración de redes se conoce como la pérdida ambigua que consiste en la incertidumbre del regreso, del reencuentro (Boss, 1999). Pero estas migraciones son inevitables y forzosas en el transcurso del desarrollo humano. Hacen parte del cambio necesario para mantener la identidad (Linares, 1996; Morin, 1994). Este proceso también actualiza al sujeto, en este proceso se desarrolla y paradójicamente, se mantiene invariante.

En los cursos de psicología y enfermería la percepción de suficiencia de amigos aumenta cuando se compara con la primera aplicación. Esta percepción se puede explicar como resultado de la re-configuración estructural de la red. A pesar que al ingresar a los cursos superiores muchos amigos de la red se dispersen geográficamente y los intereses cambien de acuerdo con los nuevos conocimientos, expectativas y relaciones llevando con el tiempo a que los amigos acaben por perder la comunicación, cabe pensar que los alumnos de estos cursos tienen recursos para rehacer este contexto de su red social-personal. Por otro lado, varios estudiantes continúan relacionándose y personas que en un primer momento fueron solamente compañeros de clase, después de un tiempo pasaron a ser consideradas como amigos.

Es significativo resaltar que los cuadrantes en donde los alumnos de los tres cursos perciben mayor insuficiencia en el número de personas e instituciones pertenecientes a sus redes sociales-personales son en el de comunidad, el cual tiene el menor peso en la estructura de la red y en el de universidad/trabajo. Como hipótesis construimos que los estudiantes que fueron a vivir en la ciudad debido a los estudios superiores, todavía no tienen conocimiento suficiente del municipio para ingresar en los espacios comunitarios. Interesa discutir aquí que son precisamente estos contextos comunitarios los que ofrecen espacios donde es posible estructurar el tiempo y en donde se obtiene de la red la satisfacción de algunas necesidades como pasatiempos y diversión, salud, espiritualidad yapoyo social (Montero, 2003).

Aunque el peso relativo de los cuadrantes de la red social-personal de los alumnos de todos los cursos se mantuvo más o menos estable, se puede apreciar cómo esta red se amplió, principalmente en el curso de enfermería. El hecho de que todos los cuadrantes hayan ganado miembrose instituciones manteniendo cada uno de ellos el peso estable en la estructura de la red permite afirmar que durante las intervenciones los estudiantes lograron ampliar la capacidad de auto-observación y de ver las relaciones que construyen en su entorno.

La red social también evalúa las funciones que esta presta a sus miembros. En seguida se presentan los porcentajes de esas funciones y el cuadrante que las presta, en cada curso. 
Tabla 11. Psicología. Porcentaje de concentración de elementos por cuadrante del mapa de redes social-personal en la primera e en la segunda aplicaciones. Primero y último mes académicos.

\begin{tabular}{|c|c|c|c|c|c|c|c|c|}
\hline & \multicolumn{2}{|c|}{ FAMILIA } & \multicolumn{2}{|c|}{ AMIGOS } & \multicolumn{2}{|c|}{ COMUNIDAD } & \multicolumn{2}{|c|}{ UNIV/TRABALHO } \\
\hline Aplicación & $1^{\mathrm{a}}$ & $2^{\mathrm{a}}$ & $1^{\mathrm{a}}$ & $2^{a}$ & $1^{\mathrm{a}}$ & $2^{\mathrm{a}}$ & $1^{\mathrm{a}}$ & $2^{\mathrm{a}}$ \\
\hline Compañía & $42,85 \%$ & $65 \%$ & $53,57 \%$ & $35 \%$ & $3,57 \%$ & $0 \%$ & $0 \%$ & $0 \%$ \\
\hline Guía Cognitivo & $82,14 \%$ & $95 \%$ & $10,71 \%$ & $5 \%$ & $0 \%$ & $0 \%$ & $0 \%$ & $0 \%$ \\
\hline Regulación Social & $71,43 \%$ & $79 \%$ & $3,57 \%$ & $15 \%$ & $0 \%$ & $0 \%$ & $0 \%$ & $0 \%$ \\
\hline Apoyo Emocional & $85,71 \%$ & $70 \%$ & $10,71 \%$ & $10 \%$ & $0 \%$ & $15 \%$ & $0 \%$ & $5 \%$ \\
\hline $\begin{array}{l}\text { Acceso a nuevos } \\
\text { contactos }\end{array}$ & $42,85 \%$ & $25 \%$ & $32,14 \%$ & $50 \%$ & $17,86 \%$ & $15 \%$ & $9,1 \%$ & $0 \%$ \\
\hline
\end{tabular}

Tabla 12. Filosofía. Porcentaje de concentración de elementos por cuadrante del mapa de redes social-personal en la primera e en la segunda aplicaciones. Primero y último mes académicos.

\begin{tabular}{lcccccccc} 
& \multicolumn{2}{c}{ FAMILIA } & \multicolumn{2}{c}{ AMIGOS } & \multicolumn{2}{c}{ COMUNIDAD } & \multicolumn{2}{c}{ UNIV/TRABALHO } \\
\hline Aplicación & 1 a & $2 \underline{a}$ & $1 \underline{a}$ & $2 \underline{a}$ & $1 \underline{a}$ & $2 \underline{a}$ & $1 \underline{a}$ & $2 \underline{a}$ \\
Compañía & $86 \%$ & $64 \%$ & $14 \%$ & $36 \%$ & $0 \%$ & $0 \%$ & $0 \%$ & $0 \%$ \\
Guía Cognitivo & $29 \%$ & $27 \%$ & $57 \%$ & $36 \%$ & $14 \%$ & $29 \%$ & $0 \%$ & $8 \%$ \\
Regulación Social & $71 \%$ & $64 \%$ & $29 \%$ & $36 \%$ & $0 \%$ & $0 \%$ & $0 \%$ & $0 \%$ \\
Apoyo Emocional & $43 \%$ & $54 \%$ & $57 \%$ & $28 \%$ & $0 \%$ & $18 \%$ & $0 \%$ & $0 \%$ \\
Acceso a nuevos & & & & & & & & \\
Contactos & $0 \%$ & $27 \%$ & $57 \%$ & $36 \%$ & $14 \%$ & $19 \%$ & $29 \%$ & $18 \%$ \\
\hline
\end{tabular}

Tabla 13. Enfermería. Porcentaje de concentración de elementos por cuadrante del mapa de redes social-personal en la primera e en la segunda aplicaciones. Primero y último mes académicos.

\begin{tabular}{|c|c|c|c|c|c|c|c|c|}
\hline & \multicolumn{2}{|c|}{ FAMILIA } & \multicolumn{2}{|c|}{ AMIGOS } & \multicolumn{2}{|c|}{ COMUNIDAD } & \multicolumn{2}{|c|}{ UNIV/TRABALHO } \\
\hline Aplicación & $1 \underline{a}$ & $2^{a}$ & $1 \underline{a}$ & 2 & $1 \underline{a}$ & $2 \underline{a}$ & $1 \underline{a}$ & $2^{a}$ \\
\hline Compañía & $62,5 \%$ & $57 \%$ & $37,5 \%$ & $43 \%$ & $0 \%$ & $0 \%$ & $0 \%$ & $0 \%$ \\
\hline Guía Cognitivo & $62,5 \%$ & $95 \%$ & $31,25 \%$ & $5 \%$ & $6,25 \%$ & $0 \%$ & $0 \%$ & $0 \%$ \\
\hline Regulación Social & $56,25 \%$ & $81 \%$ & $43,75 \%$ & $19 \%$ & $0 \%$ & $0 \%$ & $0 \%$ & $0 \%$ \\
\hline Apoyo Emocional & $50 \%$ & $57 \%$ & $25 \%$ & $14 \%$ & $0 \%$ & $5 \%$ & $6,25 \%$ & $5 \%$ \\
\hline $\begin{array}{l}\text { Acceso a nuevos } \\
\text { contactos }\end{array}$ & $12,5 \%$ & $24 \%$ & $62,5 \%$ & $47 \%$ & $6,25 \%$ & $19 \%$ & $18,75 \%$ & $5 \%$ \\
\hline
\end{tabular}


Sobre las funciones de la red, estas son, según LópezCananas (1997) y Sluzki (1997), Compañía que es cuando una persona necesita sentirse acompañada para pasar el tiempo; Guía Cognitivo, cuyo papel es transmitir la cultura, costumbres y valores de un determinado contexto cultural; Regulación Social es la función que permite establecer límites y ejerce el papel de mediador de conflictos; Apoyo Emocional es característico de relaciones íntimas y ofrece apoyo emocional. en esta forma de relación la persona puede mostrar de sí lo que siente profundamente y, por último Acceso a Nuevos Contactos, que ser trata de la posibilidad que ofrecen determinados elementos de la red para conectar con nuevos miembros, permitiendo, de esta forma, ampliar la red social-personal.

Compañía: esta función disminuyó en el cuadrante de la familia en la segunda aplicación del mapa y aumentó en el cuadrante de amigos en los cursos de filosofía enfermería (tablas 12 y 13). Se puede entender esta disminución y amento por distancia geográfica de las familias y porque los amigos son las personas con quienes pasan mayor tiempo juntos. Sin embargo, en el curso de psicología (tabla 11), la función de compañía se solicita más en el cuadrante de la familia, también en la segunda aplicación del mapa. Los estudiantes pasan a entender la familia de otro modo. Las expectativas sobre las relaciones y el comportamiento de los discentes de este curso favorecieron este cambio perceptivo y relacional, lo que apareció en la discusión de los grupos focales realizados con los mismos.

Guía cognitivo: esta función presenta incremento en la segunda aplicación en el cuadrante de la familia y disminución considerable en el cuadrante de los amigos en el curso de enfermería, estando ausente en el cuadrante de universidad/trabajo y manteniéndose de esa forma en las dos aplicaciones. La hipótesis que construimos es que al alejarse físicamente de la familia, esta pasa a ocupar un lugar importante en la percepción del lugar que ocupa en la red, probablemente porque los estudiantes al distanciarse perciben de otra forma sus relaciones familiares. Además, esta función no requiere de la presencia directa quien la ofrece, como la función anterior en que si es necesaria esta presencia. Los discentes buscan a la familia para comprender mejor el nuevo contexto en el cual están inseridos, aspecto que se incrementó con el transcurso del tiempo. Según Gimeno (1999), en el ciclo de vida familiar, cuando el adolescente sale de casa para constituirse en adulto joven, comienza a relacionarse con los padres de otra manera, considerándolos como iguales y consejeros más que como la figura anterior, a la que tenían que oponerse.

De otro lado, los discentes del curso de filosofía distribuyen la función de guía cognitivo y consejero en todos los cuadrantes de su red social-personal, ocupando la puntuación más alta el de los amigos, seguido por los cuadrantes de la familia y la comunidad. Particularmente tres cuartas partes de los estudiantes de este curso viven juntos, lo que favorece establecer vínculos de amistad. Por otro lado las características de la vivienda (seminario) influencian la percepción de la comunidad como fuente de aculturación, entendida como la transmisión de cultura, costumbre y valores (López-Cabanas, 1994; Sluzki, 1997).

Esta misma característica fue encintrada en las funciones de Regulación socialy Apoyo emocional en los cuadrantes de la familia, amigos y universidad-trabajo. Se observó que con el paso del tiempo es en la familia donde los estudiantes solicitan estas funciones de la red. Es posible pensar que las mismas explicaciones propuestas para entender el comportamiento respecto a la función de guía cognitivo, puedan utilizarse para comprender el modo en que se presenta esta característica. No obstante, se observa diferencia en el cuadrante de la comunidad para la función de Apoyo emocional, que aumentó en la segunda aplicación. Podemos pensar que los alumnos comenzaron a reconocer algunos participantes de la comunidad a los cuales pueden apelar. Un caso puede ser el PAPU*, proyecto desa-

* Programa de atención psicológica universitario, ofrecido a los estudiantes de la Universidad, a través de practicantes y profesores. 
rrollado por la Clínica Escuela de la universidad y al cual tienen acceso todos los alumnos de la institución. De hecho, se sugirió a algunos estudiantes la posibilidad de entrar en contacto con este proyecto, cuando se observó la necesidad de que algún estudiante tuviera un acompañamiento individual para cualificar su proceso de adaptación universitaria.

Acceso a nuevos contactos: esta función se incrementa en la familia y en la comunidad en la segunda aplicación del Mapa de Redes y disminuye en los cuadrantes de amigos y universidad-trabajo. Las relaciones familiares parecen mejorar globalmente; también esta función como todas las demás, es solicitada a la familia de modo más frecuente en la segunda aplicación. Igualmente podemos pensar que los estudiantes universitarios se acercan a la comunidad a través de la cual conocen otras personas e instituciones.

Ayuda de recursos y servicios: esta función aumenta en la segunda aplicación en los cuadrantes de familia y universidad-trabajo, disminuyendo en los cuadrantes de amigos y de comunidad. De esta manera observamos que la cercanía con los compañeros de la universidad acciona el poder ejercer esta función, siendo con estos con quien pasan la mayor parte del tiempo.

Así, con relación a las funciones de la red social personal de los estudiantes de los tres cursos investigados, se observó que la familia adquiere relevancia en el transcurso del primer año universitario. Pensamos también a partir de la psicología del desarrollo humano, que en este periodo evolutivo, la fase de rebeldía comienza a superarse y los valores transmitidos por la familia van siendo introyectados por el adolescente tardío y por el adulto joven (Papalia, 2005), facilitando el reracionamiento familiar y permitiendo establecer relaciones mas adultas en el contexto inmediato. Por otro lado, la universidad exige de los estudiantes comportamientos independientes, toda vez que ya no tienen profesores encargados de cuidar si asisten a las clases, por ejemplo. Cabe a ellos la responsabilidad de su presencia así como por su estudio y relaciones.

\section{Reflexiones}

En las discusiones para comprender junto con los discentes de los diversos cursos, tanto la estructura como las funciones de sus redes personales-sociales, emergieron otras temáticas que son importantes para estos y que influenciaron el proceso de transición para el ingreso en la universidad ysu proceso de aculturación. Los estudiantes del curso de psicología están preocupados por la estructuración del tiempo, sienten que no dan cuenta y se cobran cuando realizan actividades de tiempo libre o cuando se dedican al ocio en este tiempo. Esta percepción de no dar cuenta de una de las capacidades básicas del estudiante (Buendía, 1998) incremente el estrés en los universitarios. La discusión grupal y el percibir que los compañeros comparten esos pensamientos u sentimientos, posibilitó que se autoorganizaran y, durante los grupos focales para la discusión, pensaron otros modos de reestructurar las actividades para cumplir con sus deberes sin dejar de lado las actividades de diversión y ocio.

Uno de los temas repetitivos del curso de filosofía fue la percepción de que son diferentes de los otros estudiantes de la Universidad. Se sienten excluidos y observados por los compañeros de universidad. Esta sensación llevó a estos discentes a se cerrar un su grupo, creando una fuerte red interna. La cohesión podría ser la característica principal del mismo. Esta unión grupal es compartida en el ámbito externo a la universidad, especialmente por el hecho de que la mayoría vive juntos y compartir otros espacios de formación.

Los universitarios del curso de enfermería manifestaron la dificultad de vivir alejados de la familia. Los fines de semana los pasan en sus casas familiares y al regresar, los lunes, tienen deseos de desistir de los estudios. Entre ellos discutieron alternativas para superar esta situación.

Por otro lado, es interesante proponer algunas preguntas relacionadas con las características internas de los cursos, para pensar cómo estos son productores de la cultura y que podrían caracterizar aspectos particulares 
de cada uno de ellos. Por ejemplo, la preocupación por la estructuración del tiempo en el curso de psicología tendría que ver con la necesidad de ocuparse, que tiene el profesional? Y en el filosofía, sentirse diferente será un reflejo de pensarse diferente de la cultura mayoritaria, que se orienta por el hacer y tener, mas que para pensar y reflexionar sobre los asuntos del hombre y de la vida?Y, finalmente, en el curso de enfermería, la preocupación por el sanitarismo y la salud aún en ausencia de... enfermedad, llevaría a estos discentes a rechazar eventos como el ritual de primíparos por considerarlo demasiado peligroso?

Pudo observarse en los encuentros con los universitarios que la transición que sucede durante el primer año concuerda con las teorías sobre aculturación (Berry, 1995) cuando, al cambiar de contexto, muchas veces las personas vivencian un choque cultural, lo que fue confirmados en las verbalizaciones. Dijeron que el ingreso a la universidad es estresante porque además de experienciar cambios en la cultura escolar (transición del colegio para la universidad), pasan también por un periodo de alejamiento físico de sus familias por la obligación de vivir en otra ciudad; se enfrentan con la necesidad de reorganización y reconstrucción de la red social-personal, teniendo que adaptarse a las costumbres de otras micro culturas (lo que acaece al tener que conocer otra ciudad). Esta transición ecológica y los cambios consecuentes de esta llevan al estudiante a vivenciar situaciones muchas veces desconocidas y este, no teniendo herramientas apropiadas que le permitan dar cuenta de las reacciones emocionales y comportamentales derivadas, muchas veces se ve perjudicado en su vida académica.

Se percibió que el apoyo social permite que una persona construya su red de relaciones y favorezca su identidad y desempeño. Se observa en las verbalizaciones: todo el grupo está más aliviado, estamos más tranquilos, menos tensos, el grupo está mas colaborador (...) Están preguntando a los profesores cuales son los temas prioritarios, se organizan junto a los mismos para las lecturas de los textos, tenemos mas libertad, los pro- fesores también están más colaboradores con el grupo, el grupo se dio cuenta que cada uno de los compañeros maduró desde el inicio de la carrera (Psicología).

Se percibió, en los grupos focales para discusión, que los universitarios hicieron varias reflexiones a partir de las actividades propuestas. Creemos que, sus expresiones, verbalizaciones y participaciones en la actividad hayan sido productivas y reflexivas para cada uno de ellos, pues tuvieron la oportunidad de expresar sus satisfacciones e insatisfacciones respecto a la institución, a la recepción desagradable de los compañeros veteranos y entre ellos como grupo.

Esto pudo ser corroborado cuando se les preguntó: usted cambió?Yqué cambió en relación al primer semestre en la red: familia - universidad- amigos y comunidad? Respondieron que cambiaron bastante, pues al principio estaban mas precavidos, menos críticos, aceptaban lo que les era impuesto sin ningún cuestionamiento; hoy se sienten más fortalecidos para discutir situaciones o cosas que ocurrieron. Cuando se les preguntó sobre qué podrían hacer ellos para percibir mejor esta red en sus cuatro cuadrantes, dijeron que las actividades desarrolladas para propiciar las discusiones proporcionaron la posibilidad de que parasen para pensar sobre su proceso de transición ecológica, que al construir el instrumento de Mapa de Redes, al final del año lectivo, pensando sobre sus relaciones en todos los contextos, se dieron cuenta que la misma se amplió.

La universidad, cuando crea espacios extraacadémicos facilita en los estudiantes el proceso de aculturación, abre espacios para la discusión y para que emerjan propuestas alternativas diferentes a la cultura establecida y naturalizada, como fue el caso de re-pensar la recepción a los primíparos.

A pesar de que todos los alumnos del primero y del segundo semestres de los cursos investigados no haber participado de todos los encuentros, se resalta la importancia de que estos hayan sido de carácter optativo y 
no de obligación ya que, si fuera de esa manera, descaracterizaría la libertad a la participación e a la cocreación de conocimiento.

Puede afirmarse que la investigación, por lo relatado por los grupos durante los diversos encuentros, contribuyó para la inserción, inclusión y adaptación sociocultural de estos. Proporcionó espacios para la reflexión constante de sí mismos llevándolos a ser más reflexivos como sujetos sociales, ciudadanos responsables y conscientes de sus acciones. Consideramos que, de esta forma, la investigación se vuelve una herramienta de emancipación y un espacio para el desarrollo personal. La investigación activa, en la cual todos somos actores, da cuenta de las necesidades de cada una de las partes involucradas en el acto de investigar y de la construcción de conocimiento, al tiempo que los participantes dan retorno inmediato a través de su colaboración con el investigador.

Sobre este estudio, pensamos que es necesario continuar realizando aplicaciones del Mapa de Redes para observar y evaluar como se comporta la red social-personal de estudiantes universitarios y si las características que fueron observadas en los cursos de esta universidad son similares a los mismos cursos de otras universidades.

\section{Referencias}

Abengózar, M. C.; Cerdá, C. \& Meléndez, J. C. (1998). Desarrollo familiar. Aspectos teóricos y prácticos. Valencia: Promolibro.

Bajtin, M. \& Vygotsky, L. S. (1993). La organización semiótica de la conciencia. Barcelona: Anthropos.

Bauman, Z. (2005). El amor líquido. Acerca de la fragilidad de los vínculos humanos. México: Fondo de Cultura Económica.

Berger, P. \& Luckman, T. (1998). A construção social da realidade. Petrópolis: Vozes.
Berry, J. W. (1995). Psychology of aculturation. Em N. R. Goldberger \& J. B. Veroff (Eds.), The culture and psychology reader (pp. 457-488). New York: New York University Press.

Boss, P. (1999). Ambiguous loss: learning to live with unresolved grief. Cambridge: Harvard University Press.

Brenson, G. (1980). El reino de lo nuestro. Bogotá: Tercer Mundo Editores.

Buendía, H. (1998). Educación. La agenda del siglo XXI. Bogotá: Tercer Mundo Editores.

Caplan (1974). In López-Cabanas, M. \& Chacón, F (1997). Intervención psicosocial y servicios sociales. Madrid: Síntesis Psicológica.

Castel, R. (1995). ¿Qué significa estar protegido? In Dabas, E. \& Najmanovich, D (comps.) (1995). Redes. El lenguaje de los vínculos (pp. 151-158). Argentina: Paidós.

Cobb (1997). Em López-Cabanas, M. \& Chacón, F (1997). Intervención psicosocial y servicios sociales - Un enfoque participativo. Madrid: Síntesis Psicológica.

Delgado J. \& Gutiérrez, J. (1995). Métodos y técnicas cualitativas de investigación en ciencias sociales. España: Síntesis Psicología.

Elkaïm, M. (1995). Las prácticas de la terapia de red. Barcelona: Gedisa.

Foerster, L. Von (1960). Self-Organizing Systems. Yovitz and Cameron. In Ibáñez, J (1998). Nuevos avances en la investigación social II. España: Proyecto A.

Gimeno, A. (1999). La familia: el desafío de la diversidad. Barcelona: Editorial Ariel.

Gracia, E. (1998). El apoyo social en la intervención comunitaria. Barcelona: Paidós. 
Guareschi, P. (1996). A técnica dos grupos focais. PósGraduação em Psicologia, PUCRS, Porto Alegre.

Ibáñez, J. (1998). Nuevos avances en la investigación social II. España: Proyecto A.

IBGE (2006). Instituto Brasileiro de Pesquisa. Acessado em outubro de 2006. Disponível em: http: // www.ibge.gov.br/cidadesat/default.php

Johnston, J. (1973). Lesbian nation: the feminist solution. New York: Simon and Schuster.

Lin, Dean \&Ensel (1986). En López-Cabanas, M. \&Chacón, F. (1997). Intervención psicosocial y servicios sociales. Madrid: Síntesis Psicológica.

Linares, J. L. (1996). Identidad y narrativa. Barcelona: Paidós.

López-Cabanas, M, \& Chacón, F. (1997). Apoyo social, redes sociales e grupos de autoayuda. En LópezCabanas, M. \& Chacón, F. (1997). Intervención psicosocial y servicios sociales. Madrid: Síntesis Psicológica.

McGoldrick, M. (2003). Novas abordagens em terapia familiar. Raça cultura e gênero na prática clínica. São Paulo: Roca.

Montero, M. (2003). Teoría y práctica de la psicología comunitaria: la tensión entre la sociedad y la comunidad. Buenos Aires: Paidós.

Morgan, D. L. (1988). Focus groups as qualitative research. Newbury Park CA: Sage Publication.
Morin, E. (1994). La noción del sujeto. En Fried, D. (1994). Nuevos paradigmas, cultura y subjetividad. Buenos Aires: Paidós.

Papalia, D. (2005). Desenvolvimento humano. Porto Alegre: Artmed.

Rangel, M. P. (2003). Família imigrante: estrutura e funcionamento da rede social. Dissertação de mestrado. Faculdade de Psicologia, PUCRS.

Rangel, M. P. (2004). A construção das redes sócio-familiares e a orientação aculturativa de famílias imigrantes hispano-americanas. Projeto para seleção de doutorado PUC-RS.

Rangel, M. P. \& Sarriera, J (2005). Redes sociais na investigação psicossocial. Em Aletheia, 21, jan-jun, p. 53-68.

Romero, S. M. (2000). A Utilização da metodologia dos grupos focais na pesquisa em psicologia. Em Scarparo, H. (2000). Psicologia e pesquisa - perspectivas metodológicas. Porto Alegre: Sulina.

Sluzki, C. (1997). A rede social na prática sistêmica. Alternativas terapêuticas. São Paulo: Casa do Psicólogo.

Thompson, J. B. (1995). Ideologia e cultura moderna: teria social crítica na era dos médios de comunicação de massa. Petrópolis: Vozes.

Wasserman, H. \& Danforth, H. (1988). The human bond: support groups and mutual aid. New York: Springer Publishing Company. 